\title{
“Como ser um líder positivo?”: relações entre estilos de lideranças e forças de caráter
}

\author{
"How to be a positive leader?": relationships between leadership styles and characther strenghts \\ “Cómo ser un líder positivo?”: relaciones entre estilos de liderazgo y fuerzas del carácter
}

Recebido: 27/12/2021 | Revisado: 01/01/2022 | Aceito: 07/01/2022 | Publicado: 10/01/2022

\author{
Juliana Taglhare Garcia \\ ORCID: https://orcid.org/0000-0001-8313-4466 \\ Universidade São Francisco, Brasil \\ E-mail: jtaglhare@mxb.com.br \\ Ana Paula Porto Noronha \\ ORCID: https://orcid.org/0000-0001-6821-0299 \\ Universidade São Francisco, Brasil \\ E-mail: ana.noronha8@gmail.com \\ Leonardo de Oliveira Barros \\ ORCID: https://orcid.org/0000-0002-8406-0515 \\ Universidade Federal da Bahia, Brasil \\ E-mail: leonardobarros_lob@hotmail.com
}

\begin{abstract}
Resumo
Os estudos no campo do trabalho são geralmente conduzidos com colaboradores que não estão em posições de liderança, tornando importante investigações que contemplem líderes e com ênfase na promoção de ações positivas e virtuosas. O objetivo principal deste estudo foi analisar as relações entre forças de caráter e estilos de liderança. Participaram 150 líderes de empresas de São Paulo e Minas Gerais, sendo 54,7\% do sexo masculino e atuando na função por um período médio de 9,47 anos. Como instrumentos foram utilizados um questionário demográfico, a Escala de Forças de Caráter e a Escala de Avaliação do Estilo Gerencial. Os resultados indicaram que as forças de caráter estavam mais correlacionadas ao fator relacionamento. Além disso, por meio da path analysis, as forças de caráter explicaram positivamente o fator relacionamento e negativamente o fator tarefa dos estilos gerenciais. Os resultados são discutidos à luz da literatura e dão indicativos de como as forças de caráter podem ser utilizadas em intervenções com líderes.
\end{abstract}

Palavras-chave: Psicologia positiva; Trabalho; Cultura organizacional.

\begin{abstract}
Studies in the field of work are generally conducted with employees who are not in leadership positions, making important investigations that include leaders and with an emphasis on promoting positive and virtuous actions. The main objective of this study was to analyze the relationships between character strengths and leadership styles. 150 business leaders from companies in São Paulo and Minas Gerais participated, 54.7\% of whom were male and worked in the function for an average period of 9.47 years. As instruments, a demographic questionnaire, the Escala de Forças de Caráter and the Escala de Avaliação do Estilo Gerencial were used. The results indicated that the character strengths were more correlated to the relationship factor. In addition, through path analysis, the character strenghts explained positively the relationship factor and negatively the task factor of managerial styles. The results are discussed in light of the literature and give indications of how character strengths can be used in interventions with leaders.
\end{abstract}

Keywords: Positive psychology; Work; Organizational culture.

\section{Resumen}

Los estudios en el campo de trabajo generalmente se realizan con empleados que no están en posiciones de liderazgo, haciendo importantes las investigaciones que incluyen a los líderes y con énfasis en promover acciones positivas y virtuosas. El objetivo principal de este estudio fue analizar las relaciones entre las fuerzas del carácter y los estilos de liderazgo. Participaron 150 líderes empresariales de São Paulo y Minas Gerais, de las cuales el 54,7\% eran hombres y trabajaron en la función durante un período promedio de 9,47 años. Como instrumentos, se utilizaron un cuestionario demográfico, a Escala de Forças de Caráter e a Escala de Avaliaão do Estilo Gerencial. Los resultados indicaron que las fuerzas del carácter estaban más correlacionadas con el factor de relación. Además, a través del análisis de ruta, las fuerzas de carácter explicaron positivamente el factor de relación y negativamente el factor de tarea de los estilos gerenciales. Los resultados se discuten a la luz de la literatura y dan indicaciones de cómo se pueden utilizar las fortalezas del carácter en intervenciones con líderes.

Palabras clave: Psicología positiva; Trabajo; Cultura organizacional. 


\section{Introdução}

A Psicologia Positiva tem como objetivo realizar estudos e intervenções centradas na promoção de experiências humanas que gerem bem-estar subjetivo (Peterson, 2013; Reppold et al., 2019). Um dos conceitos centrais da Psicologia Positiva refere-se às forças de caráter/pessoais, definidas como a capacidade pré-existente para que uma forma particular de comportamento, pensamento ou sentimento se apresente de maneira autêntica para o indivíduo e lhe permita um funcionamento saudável (Noronha \& Reppold, 2019; Peterson \& Seligman, 2004). Quanto mais as forças estiverem presentes nos indivíduos, maiores serão as chances de vivência de mais emoções positivas, melhores relacionamentos interpessoais e maior engajamento em atividades como o trabalho (Littman-Ovadia et al., 2017; Seligman, 2009). As forças de caráter/pessoais são também entendidas como traços de personalidade, com valor moral e que podem ser aprendidas e construídas sobre padrões consensuais ou mesmo por modelos destacados pela cultura na qual o indivíduo está inserido (Peterson \& Park, 2006).

As investigações acerca das forças de caráter/pessoais são frequentes na literatura, abordando contextos e públicos diversos, sobretudo no contexto estrangeiro (Lavy, 2019; Noronha, \& Batista, 2020; Tehranchi et al., 2018; Yan, Chan, Chow, Zheng \& Sun, 2020). No entanto, em relação ao contexto laboral, os estudos têm buscado analisar a relação entre as forças e variáveis como a satisfação com o trabalho (Heintz \& Ruch, 2019), comportamento pró-social (Freidlin \& Littman-Ovadia, 2019), performance no trabalho (Pang \& Ruch, 2019), fator protetivo para a saúde mental do trabalhador (Kim et al., 2018), entre outros. Tal como destacado por Vazquez et al. (2019), a Psicologia Positiva voltada à gestão de pessoas tem como premissa a compreensão de que a energia laboral associada ao lazer promove desenvolvimento saudável aliado à produção. Destaca-se que, em geral, os estudos no âmbito do trabalho são realizados com os colaboradores que não ocupam cargos de chefia ou liderança das equipes, indicando que as pesquisas sobre liderança e organizações positivas ainda são iniciais no cenário nacional (Adriano et al., 2018).

A investigação sobre os níveis de forças de caráter de líderes é importante para que os estudos das organizações positivas contemplem uma quantidade maior de agentes do espaço organizacional. Considerando que a liderança tem ocupado um lugar nuclear nos contextos de trabalho, um importante desafio passa a ser o de capacitar as organizações e seus líderes que não se mostram seguros de suas capacidades para liderar (Uhl-Bien \& Arena, 2017), pois é possível desenvolver tais competências ao longo da vida e nos contextos de trabalho (Northouse, 2018).

Neste estudo a liderança é entendida como situacional, a partir da inter-relação entre o direcionamento oferecido pelo líder, a quantidade de apoio emocional e o nível de desenvolvimento dos subordinados em relação à tarefa (Melo, 2014). Em termos específicos, uma liderança positiva é aquela na qual há a valorização das forças de caráter em detrimento das fraquezas, promovendo o bem-estar e engajamento no trabalho ao mesmo tempo em que encoraja os liderados no alcance dos resultados (Cameron, 2013). De tal modo, uma liderança virtuosa e autêntica pauta-se na autoconsciência, na valorização de relacionamentos de qualidade entre líder e liderados, no desenvolvimento de potencialidades sem deixar de lado os interesses econômicos das organizações (Campos \& Rueda, 2018). Ao mesmo tempo, líderes positivos têm ações consistentes e preocupam-se em promover a justiça, honestidade, coragem e a prudência (Guimarães et al., 2019).

O estilo de liderança influência o desenvolvimento das equipes e organizações (Cameron, 2013), sendo que na perspectiva de Melo (2014) estes podem ser de três tipos. O primeiro estilo é do líder voltado para tarefa, marcado pelo autoritarismo, dominância, persuasão, designação de metas e supervisão de comportamentos. Um segundo estilo refere-se ao líder voltado para os relacionamentos, no qual há ações mais democráticas, confiança mútua, valorização das relações interpessoais na busca de satisfação dos membros do grupo. Por fim, o último estilo é o do líder voltado para a situação, que implica na habilidade de identificar as mudanças necessárias em seu ambiente de trabalho e adaptar seu estilo às exigências deste ambiente de acordo com o nível de desenvolvimento e capacidade de execução de tarefas dos seus liderados. 
As pesquisas acerca das relações entre forças de caráter e liderança indicam que a valorização e aplicação das forças no trabalho melhoram o desempenho dos líderes, impactando na forma como influenciam pessoas e no alcance das metas propostas (Garcea et al., 2012). Neste sentido, o desenvolvimento das forças pode contribuir para a formação de equipes e na execução de um trabalho de qualidade (Vianna, 2016), bem como, ser útil no momento de recrutar e selecionar novos colaboradores para a organização (Liney \& Garcea, 2013). Os níveis das forças de caráter dos líderes também devem ser considerados no momento da atribuição do cargo, uma vez que apenas competências técnicas não significam adequação para o exercício das funções (Cunha \& Rego, 2015).

Assim, estar em posição de liderança não confere ao indivíduo as competências e habilidades necessárias para liderar uma equipe (Uhl-Bien \& Arena, 2017). A prática da liderança depende também da existência da liderança enquanto qualidade pessoal, sendo que esta é caracterizada pela motivação e capacidade de buscar, atingir e desempenhar com sucesso o papel de líder nos sistemas sociais (Peterson \& Seligman, 2004). Para os autores, a liderança é uma força de caráter/pessoal e pode ser desenvolvida. Mais especialmente, o indivíduo que pontua alto na força possui características para influenciar e ajudar os outros, assim como, fortalecer ações na direção do sucesso coletivo. Coordenar atividades, criar e desenvolver boas relações entre os integrantes de um grupo, organizar sistemas sociais de forma bem-sucedida são alguns exemplos de ações que podem ser efetivadas pelas pessoas que têm a força (Noronha, \& Reppold, 2019).

Frente a isso, compreendendo que as forças de caráter podem contribuir para o desenvolvimento da liderança enquanto prática laboral e qualidade pessoal, este estudo teve por objetivo principal analisar as relações entre forças e caráter e estilos de liderança. Além disso, buscou-se verificar diferenças em função das variáveis demográficas e o potencial explicativo das forças de caráter sobre os estilos de liderança.

\section{Metodologia}

Trata-se de um estudo quantitativo, descritivo e com delineamento survey do tipo interseccional (Calais, 2007). O estudo foi realizado com uma amostra não-probabilística de conveniência.

\section{Participantes}

Participaram deste estudo 150 pessoas, que ocupavam cargos de líderes há pelo menos seis meses em empresas localizadas em São Paulo e em Minas Gerais, com idades variando entre 20 e 63 anos, $(M=38,15 ; D P=8,93)$, sendo 54,7\% ( $n$ = 82) do sexo masculino. Em relação ao nível de escolaridade, 46\% ( $\mathrm{n}=69)$ declarou ter cursado pós-graduação, 38\% $(\mathrm{n}=57)$ o ensino superior e $16 \%(\mathrm{n}=24)$ o ensino médio. Os participantes atuavam na função há um tempo variando de 1 a 40 anos $(\mathrm{M}$ = 9,47; $\mathrm{DP}=7,87)$ e com número de subordinados diretos entre 1 e $120(\mathrm{M}=9,63$; $\mathrm{DP}=12,91)$. As empresas nas quais trabalhavam apresentavam entre 4 a 100.000 funcionários, sendo predominantemente nas áreas de serviços $(25,3 \% ; n=38)$, têxtil $(20 \% ; \mathrm{n}=30)$ e financeiro $(17,3 \% ; \mathrm{n}=26)$.

\section{Instrumentos}

Questionário de identificação (elaborado pelos autores): teve como objetivo buscar informações de variáveis sociodemográficas e contextuais, tais como idade, sexo, escolaridade, tempo de atuação na função, número de reportes imediatos (subordinados), número de funcionários e ramo da empresa.

Escala de Forças de Caráter - EFC (Noronha \& Barbosa, 2016): composta por 71 itens respondidos em escala Likert, variando de zero (nada a ver comigo) a 4 (tudo a ver comigo). O objetivo do instrumento é investigar as 24 forças de caráter propostas por Peterson e Seligman (2004), sendo que cada força é representada por três itens, com exceção da força 
Apreciação ao Belo que é composta por dois itens apenas. O índice de precisão da escala em sua versão de construção foi de 0,93 .

Escala de Avaliação do Estilo Gerencial - EAEG (Melo, 2014): composta por 19 itens respondidos em escala Likert, variando de 1 (nunca age assim) a 5 (sempre age assim). O objetivo deste instrumento é identificar se o estilo do líder é voltado para tarefa (6 itens), relacionamento ( 9 itens) ou situacional (4 itens). A escala originalmente é aplicada às pessoas que tenham relacionamentos funcionais com seus líderes, descrevendo comportamentos apresentados pelas lideranças no dia a dia de trabalho com os seus subordinados, porém, para esta pesquisa foi aplicada no próprio líder, de modo que a instrução da aplicação foi alterada para "como você se comporta no dia a dia" e a chave de resposta para 1 (nunca ajo assim) a 5 (sempre ajo assim). Em relação à precisão, em sua versão de construção o instrumento apresentou alfa de Cronbach de 0,94 no fator relacionamento, 0,72 no fator tarefa e 0,82 no fator situacional. No presente estudo, considerando a adaptação nos descritores da chave de resposta, os índices (alfa de Cronbach) foram de 0,79 no fator relacionamento, 068 no fator tarefa e 0,60 no fator situacional.

\section{Procedimentos}

A pesquisa foi aprovada pelo Comitê de Ética em Pesquisa da Universidade São Francisco (CAAE 62364716.7.0000.5514). Os participantes foram convidados por meio de correio eletrônico, sendo que após o aceite houve o agendamento para aplicação dos instrumentos de modo individual e na própria empresa em que atuavam. Os respondentes assinaram o Termo de Consentimento Livre Esclarecido e na sequência responderam ao questionário de identificação, a EFC e a EAEG em um tempo aproximado de 30 minutos.

\section{Análise de dados}

As estatísticas descritivas foram realizadas por meio do software SPSS (versão 23), assim como as comparações de médias (teste $\mathrm{t}$ de Student e análise de variância -ANOVA - com post hoc de Tukey) dos fatores em função das variáveis demográficas, a análise de correlação de Pearson entre as forças de caráter e estilos de lideranças e os índices de confiabilidade da EAEG. A path analysis foi realizada no software Mplus (Muthén \& Muthén, 2011) tendo como estimador o maximum likelihood robusto (MLR). Em relação aos índices de ajustes, adotaram-se os seguintes critérios: Confimatory Fit Index (CFI) e Tucker-Lewis Index (TLI) iguais ou superiores a 0,95 e Root Mean Square Error of Approximation (RMSEA) igual ou menor do que 0,08 (Hu \& Bentler, 1999).

\section{Resultados}

Inicialmente foram buscadas diferenças nas médias em função das variáveis demográficas, sendo que serão apresentados apenas os resultados estatisticamente significativos. Em relação ao sexo, observou-se que mulheres apresentaram maiores médias quando comparadas aos homens nas forças amor ao aprendizado $(t(148)=2,186, \mathrm{p}<0,05)$, autenticidade $(t(148)=2,376, \mathrm{p}<0,05)$, amor $(t(148)=2,256, \mathrm{p}<0,05)$, bondade $(t(148)=3,198, \mathrm{p}<0,05)$, inteligência social $(t(148)=3,087$, $\mathrm{p}<0,05)$, modéstia $(t(148)=2,678, \mathrm{p}<0,05)$ e gratidão $(t(148)=2,059, \mathrm{p}<0,05)$, bem como no fator relacionamento dos estilos de lideranças $(t(148)=2,126, \mathrm{p}<0,05)$.

Ao realizar a comparação em função da escolaridade, observou-se que participantes com pós-graduação completa obtiveram maiores médias nas forças criatividade $(F(2)=3,649, \mathrm{p}<0,05)$, inteligência social $(F(2)=3,257$, p $<0,05)$ e no fator relacionamento $(F(2)=3,135, \mathrm{p}<0,05)$, enquanto aqueles que cursaram até o ensino médio apresentaram maiores médias em perdão $(F(2)=3,742, \mathrm{p}<0,05)$ e no fator tarefa $(F(2)=6,346, \mathrm{p}<0,05)$. Em relação ao tempo na função, participantes com menor tempo obtiveram maiores médias na força modéstia em detrimento daqueles com maior experiência no cargo $(F(2)=3,521$, 
p <0,05). Ao analisar os dados em função do número de subordinados não foram encontrados resultados com significância estatística. O número de funcionários não foi considerado tendo em vista a discrepância na amplitude dos dados. Na sequência realizou-se a análise de correlação de Pearson entre as forças de caráter e os estilos de liderança e os resultados são apresentados na Tabela 1.

Tabela 1. Correlação entre forças de caráter e estilos de liderança.

\begin{tabular}{|c|c|c|c|}
\hline \multirow[t]{2}{*}{ Forças de caráter } & \multicolumn{3}{|c|}{ Estilos de Liderança } \\
\hline & Relacionamento & Situacional & Tarefa \\
\hline Criatividade & $0,34^{* *}$ & $0,23^{* *}$ & 0,01 \\
\hline Curiosidade & $0,21^{\text {** }}$ & $0,16^{*}$ & $0,20^{*}$ \\
\hline Pensamento Crítico & $0,27^{* *}$ & 0,15 & 0,14 \\
\hline Amor ao aprendizado & $0,28^{* *}$ & 0,08 & $0,25^{* *}$ \\
\hline Sensatez & $0,28^{* *}$ & 0,12 & $-0,01$ \\
\hline Bravura & $0,18^{*}$ & 0,14 & $0,19^{*}$ \\
\hline Perseverança & 0,15 & 0,06 & $0,35^{* *}$ \\
\hline Autenticidade & $0,19^{*}$ & 0,09 & 0,15 \\
\hline Vitalidade & 0,11 & 0,08 & 0,04 \\
\hline Amor & 0,15 & 0,09 & 0,09 \\
\hline Bondade & $0,43^{* *}$ & $0,22^{* *}$ & $0,16^{*}$ \\
\hline Inteligência Social & $0,38^{* *}$ & 0,13 & 0,05 \\
\hline Cidadania & $0,31^{\text {** }}$ & 0,15 & $0,19^{*}$ \\
\hline Imparcialidade & $0,28^{* *}$ & 0,11 & $0,17^{*}$ \\
\hline Liderança & $0,17^{*}$ & 0,13 & $0,29^{* *}$ \\
\hline Perdão & $0,16^{*}$ & 0,04 & $0,17^{*}$ \\
\hline Modéstia & $0,21^{\text {** }}$ & 0,10 & $0,18^{*}$ \\
\hline Prudência & $0,19^{*}$ & 0,03 & $0,32^{* *}$ \\
\hline Autorregulação & $0,28^{* *}$ & 0,03 & 0,06 \\
\hline Apreciação do belo & $0,26^{* *}$ & 0,11 & 0,04 \\
\hline Gratidão & $0,28^{* *}$ & 0,05 & 0,14 \\
\hline Humor & $0,23^{* *}$ & 0,14 & $-0,09$ \\
\hline Esperança & 0,07 & 0,05 & 0,11 \\
\hline Espiritualidade & $-0,06$ & $-0,09$ & 0,13 \\
\hline
\end{tabular}

Nota: $* \mathrm{p}<0,05 ; * * \mathrm{p}<0,001$. Fonte: Autores.

Observa-se que com exceção das forças vitalidade, amor, esperança e espiritualidade que não apresentaram correlações estatisticamente significativa com nenhum fator dos estilos de liderança, todas as demais correlacionaram-se em sentido positivo com um dos estilos. O maior número de correlações foi obtido para as forças de caráter e o fator relacionamento, sendo que as magnitudes dos coeficientes variaram de fraco à moderado. $\mathrm{O}$ estilo situacional foi o que apresentou a menor quantidade de correlações com as forças de caráter. Na sequência foi testado um modelo de path analysis irrestrito no intuito de verificar o potencial explicativo das forças de caráter em relação aos estilos de liderança, conforme a Figura 1. 
Figura 1. Modelo de path analysis das forças de caráter explicando os estilos gerenciais.

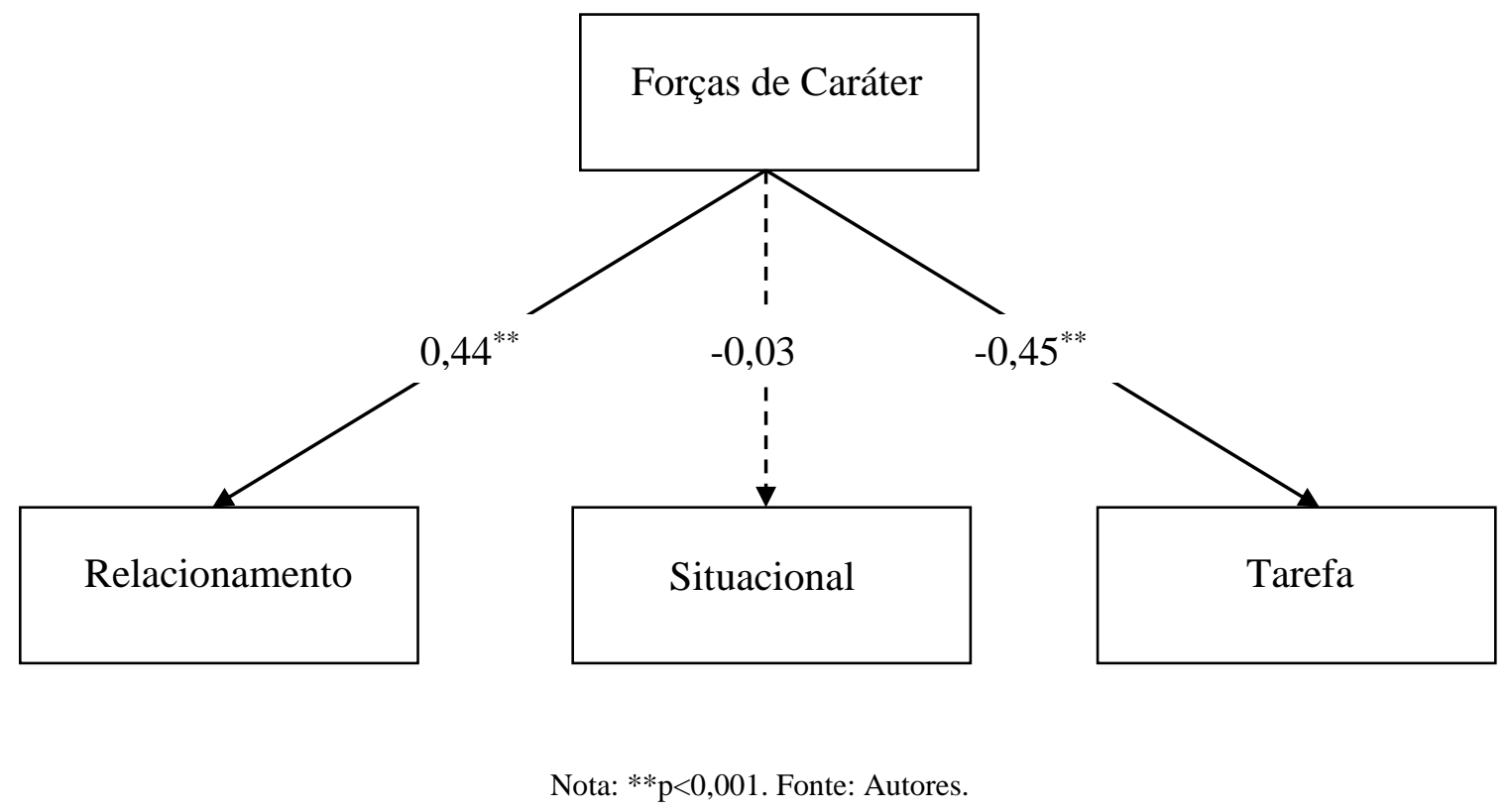

Observa-se que as forças de caráter explicam positivamente e estatisticamente significativo apenas os estilos de liderança relacionamento e situacional. Os índices de ajuste obtidos a partir do modelo foram excelentes: $\mathrm{CFI}=1,00$; TLI $=$ 1,$00 ; \operatorname{RMSEA}=0,00$. No que se refere à variância explicada $\left(R^{2}\right)$ para o fator relacionamento $0,20(p<0,001)$, situacional 0,00 $(\mathrm{p}, 0,82)$ e tarefa $0,20(\mathrm{p}<0,01)$.

\section{Discussão}

Liderança é entendida como uma força de caráter/pessoal sob a perspectiva da Psicologia Positiva, tal como preconizado por Peterson e Seligman (2004). Assim, este estudo investigou se a presença das forças, inclusive da própria liderança, favorece estilos de liderança mais voltado ao relacionamento, à tarefa ou ao situacional (Melo, 2014). Ao analisar os resultados de diferenças de médias em função do sexo, os resultados observados para as forças de caráter são comuns aos reportados na literatura, nos quais as mulheres apresentam maiores médias quando comparadas aos homens (Heintz et al., 2019; Kim, et. al, 2018; Noronha, \& Barbosa, 2016; Noronha \& Martins, 2016). De modo geral, as mulheres apresentaram maiores níveis nas forças que envolvem o cuidado, a ajuda e a ação sábia nos relacionamentos (Peterson \& Seligman, 2004). Já em relação aos estilos de liderança, o grupo feminino apresentou maiores médias no fator relacionamento, no qual há o predomínio de características relativas à valorização das relações interpessoais (Melo, 2014). Tais resultados indicam que tanto em relação às características pessoais como do estilo de liderança, as mulheres tendem a adotar ações em que se sobressaia uma forma de interação social positiva com respeito à opinião do outro.

As diferenças em função da escolaridade indicaram que participantes com maior escolaridade adotam mais o estilo de liderança pautado no relacionamento ao mesmo tempo em que apresentam maiores níveis nas forças de caráter que se referem à abertura para novas formas de executar ações e de agir sabiamente nos relacionamentos (Peterson \& Seligman, 2004). Por sua vez, os participantes com menor escolaridade tendem a apresentar maior nível de perdão enquanto adotam um estilo de liderança marcado pela designação autocrática de metas e supervisão de comportamentos (Melo, 2014). Assim, os dados sugerem que a escolaridade influencia tanto nas forças de caráter como no estilo de liderança, sendo que no presente estudo quanto maior o nível escolaridade, maior também a capacidade em adotar um estilo mais democrático no manejo das tarefas dentro do ambiente organizacional. 
Em relação aos coeficientes de correlação, observou-se que o estilo pautado nos relacionamentos foi o que mais obteve aqueles significativos com as forças de caráter. Uma vez que este estilo é caracterizado pela valorização de ações participativas, confiança mútua e satisfação das necessidades de todos (Melo, 2014), as correlações reforçam o potencial das forças de caráter em produzirem boas consequências (Littman-Ovadia, et. al, 2017; Peterson \& Seligman, 2004). De tal modo, quanto mais desenvolvidas forem as forças, maior tende a ser a capacidade do líder em influenciar as pessoas e fazer uma gestão adequada e participativa. Cabe ressaltar que este estilo não apresentou correlações com as forças que se referem ao entusiasmo, à crença de um futuro positivo e a perseverança, aspectos que podem contribuir para momentos de crise ou para a execução de tarefas mais complexas (Noronha \& Reppold, 2019).

O estilo situacional apresentou correlações significativas apenas com as forças criatividade, curiosidade e bondade. Este tipo de liderança se caracteriza por adaptações ao estilo de liderar de acordo com as demandas e níveis dos liderados (Melo, 2014), o que demanda habilidades como as oriundas das forças relacionadas. Um líder que atua de acordo com a situação precisa ter curiosidade para conhecer sua equipe, ser cuidadoso e atento ao próximo e ter criatividade na elaboração de novas formas de executar as tarefas de acordo com as características dos membros da sua equipe (Garcea et al., 2012). Por sua vez, o estilo de liderança com foco na tarefa esteve mais fortemente correlacionado às forças de caráter perseverança $\mathrm{e}$ prudência, o que é considerado pertinente dado que este tipo de líder atua planejando bem seus objetivos e metas e foca na realização deles.

A partir do modelo de path analysis foi possível perceber que as forças de caráter explicaram de modo estatisticamente significativo, positivamente e com magnitude moderada o estilo de liderança com foco no relacionamento e negativamente em magnitude moderada o estilo de tarefa. Assim, entende-se que as forças de caráter podem ajudar os líderes no ajuste de seus comportamentos com vistas ao melhor resultado possível em uma determinada situação (Garcea et al., 2012) e permitindo que estes adotem posturas mais democráticas ou que aumentem e diminuam os níveis de exigência de acordo com as necessidades do ambiente laboral (Melo, 2014). Além disso, as forças tendem a diminuir comportamentos do líder com foco apenas no desempenho de tarefas ou estilos mais autocráticos.

Cabe ressaltar que estilos de liderança que reforcem a partilha das decisões e incentive a proximidade nas relações tendem a contribuir para a atribuição de autenticidade por parte dos subordinados ao líder (Cervo et al., 2018). Assim, se a liderança está além da ocupação de um cargo e depende do desempenho de comportamentos e ações de gerenciamento da equipe (Cunha \& Rego, 2015), o desenvolvimento das forças no contexto organizacional pode contribuir para que os líderes sejam reconhecidos como tal pelos colaboradores a partir de uma relação mais próxima. Este reconhecimento tende a ser convertido em melhor desempenho da equipe e maior efetividade no alcance das metas (Gill \& Caza, 2015).

\section{Considerações Finais}

Os achados do presente estudo permitem compreender que as forças de caráter influenciam diretamente no estilo de liderança que será adotado, reforçando apontamentos anteriores sobre essa relação (Garcea et al., 2012; Vianna, 2016). De modo mais específico, a maior contribuição foi identificar que as forças de caráter bem desenvolvidas podem fazer com que o líder adote comportamentos democráticos e que valorizem o bem-estar geral de sua equipe durante o desempenho das funções e não apenas o mero alcance de metas. Em nível prático, é preciso considerar o quanto estas características positivas são bem desenvolvidas nos candidatos a vagas de liderança, dado que podem interferir na performance do líder, da equipe e da organização (Cunha \& Rego, 2015).

Os resultados do estudo demandam de parcimônia em sua generalização dado que o tamanho amostral não é representativo e que contou com participantes de uma única região brasileira. Novos estudos que contemplem uma maior diversidade de regiões são necessários, bem como com um equilíbrio amostral em relação às áreas de atuação de modo que 
seja possível verificar o quanto estas características podem interferir nos níveis de forças de caráter e quais os estilos de liderança mais frequentes por contexto. Além disso, estudos que contemplem outras variáveis positivas tais como, otimismo, felicidade, empatia, entre outros, podem ser úteis para ampliação da compreensão sobre como potencializar estilos de lideranças positivos e virtuosos e, consequentemente, sobre como tornar o ambiente laboral como um espaço de promoção de bem-estar. Todavia, os resultados apresentados neste estudo podem servir de ponto de partida para o desenvolvimento de futuras investigações e para a elaboração de treinamentos específicos para líderes tendo como foco as forças de caráter.

\section{Agradecimentos}

O presente trabalho foi realizado com apoio da Coordenação de Aperfeiçoamento de Pessoal de Nível Superior Brasil (CAPES) - Código de Financiamento 001.

\section{Referências}

Adriano, B. M., Bertoncini, I., Esper, A. J. F., \& Cunha, C. J. C. de A. (2018). Liderança e organizações positivas: uma análise bibliométrica: uma análise bibliométrica. Anais do Congresso Internacional de Conhecimento E Inovação - Ciki, 1(1). https://proceeding.ciki.ufsc.br/index.php/ciki/article/view/426

Calais, S. L. (2007). Delineamento de Levantamento ou Survey. IN M. N. Baptista \& D. C. Campos (orgs). Metodologias de Pesquisa em Ciências. LTC.

Cameron, K. S. (2013). Practicing positive leadership: tools and techniques that create extraordinary results. Barret-Koehler.

Campos, M. I., \& Rueda, F. J. M. (2018). Evolução do construto liderança autêntica: uma revisão de literatura. Revista Psicologia Organizações e Trabalho, 18(1), 291-298. 10.17652/rpot/2018.1.13473

Cervo, C. S., Natividade, J. C., Mónico, L. D. S. M., Pais, L., Santos, N. R., \& Hutz, C. S. (2018). Modelo de Liderança Autêntica: concepção teórica e evidências de validade do Authentic Leadership Questionnaire (ALQ) para o Brasil. Psychologica, 61(2), 7-29. 10.14195/1647-8606_61-2_1

Cunha, M. P., \& Rego, A. (2015). As virtudes nas organizações. Análise Psicológica, 33(4), 349-359. 10.14417/ap.1022

Freidlin, P., \& Littman-Ovadia, H. (2019). Prosocial Behavior at Work Through the Lens of Character Strengths. Frontiers in Psychology, 10: 3046. 10.3389/fpsyg.2019.03046

Garcea, N., Linley, A., Mazurkiewicz, K., \& Bailey, T. (2012). "Future female talent development". Strategic HR Review, 11(4), 199-204. $10.1108 / 14754391211234913$

Gill, A., \& Caza, A. (2015). An Investigation of Authentic Leadership's Individual and Group Influence on Follower Responses. Journal of Management, 20(10), 1-25. 10.1177/0149206314566461

Guimarães, M., Ferreira, M. C., \& Pereira, M. (2019). Evidências iniciais da escala de liderança virtuosa. Estudos de Psicologia (Campinas), 36, e170101. $10.1590 / 1982-0275201936 \mathrm{e} 170101$

Heintz, S., Kramm, C., \& Ruch, W. (2019). A meta-analysis of gender differences in character strengths and age, nation, and measure as moderators. The Journal of Positive Psychology, 14(1), 103-112. 10.1080/17439760.2017.1414297

Heintz, S., \& Ruch, W. (2019). Character Strengths and Job Satisfaction: Differential Relationships Across Occupational Groups and Adulthood. Applied Research in Quality of Life, 1-25. 10.1007/s11482-018-9691-3

Hu, L., \& Bentler, P. M. (1999). Cutoff criteria for fit indexes in covariance structure analysis: Conventional criteria versus new alternatives. Structural Equation Modeling: A Multidisciplinary Journal, 6(1), 1-55. 10.1080/10705519909540118

Kim, H. R., Kim, S. M., Hong, J. S., Han, D. H., Yoo, S. K., Min, K. J., \& Lee, Y. S. (2018). Character strengths as protective factors against depression and suicidality among male and female employees. BMC public health, 18(1), 1084. 10.1186/s12889-018-5997-1

Lavy, S. (2019). A review of character strengths interventions in twenty-first-century schools: Their importance and how they can be fostered. Applied Research in Quality of Life, 1-24. 10.1007/s11482-018-9700-6

Linley, A., \& Garcea, N. (2013). "Engaging graduates to recruit the best". Strategic HR Review, 12(6), 297-301. 10.1108/SHR-05-2013-0050

Littman-Ovadia, H., Lavy, S., \& Boiman-Meshita, M. (2017). When theory and research collide: examining correlates of signature strengths use at work. Journal of Happiness Studies, 18(2), 527-548. 10.1007/s10902-016-9739-8

Melo, E. A. A. (2014). Liderança gerencial. In M. M. M. Siqueira (Org.). Novas medidas do comportamento organizacional: ferramentas de diagnóstico e de gestão (pp. 217-229). Artmed.

Muthén, L. K., \& Muthén, B. O. (2011). Mplus user’s guide. Sixth edition. Muthén \& Muthén. 
Research, Society and Development, v. 11, n. 1, e43011125158, 2022

(CC BY 4.0) | ISSN 2525-3409 | DOI: http://dx.doi.org/10.33448/rsd-v11i1.25158

Noronha, A. P. P., \& Barbosa, A. J. G. (2016). Forças e virtudes: escala de forças de caráter. In C. S. Hutz (2016). Avaliação em psicologia positiva: técnicas e medidas (pp. 21-43). Hogrefre.

Noronha, A. P. P., \& Batista, H. H. V. (2020). Análise da estrutura interna da Escala de Forças de Caráter. Ciencias Psicológicas, 14(1), e-2150. 10.22235/cp.v14i1.2150

Noronha, A. P. P., \& Martins, D. F. (2016). Associações entre forças de caráter e satisfação com a vida: Estudo com universitários. Actas Colombianas de Psicologia, 19(2): 97-103. 10.14718/ACP.2016.19.2.5

Noronha, A. P. P., \& Reppold, C. T. (2019). Introdução às forças de Caráter. In M. N. Baptista, M. Muniz, C. T. Reppold, C. H. S. S. Nunes, L. F. Carvalho, R. Primi, A. P. P. Noronha, A. G. Seabra, S. M. Wechsler, C. S. Hutz, \& L. Pasquali (2019). Compêndio de Avaliação Psicológica (pp xx-yy). Petrópolis: Vozes.

Northouse, P.G. (2018). Leadership: Theory and practice (8th ed.). Sage.

Pang, D., \& Ruch, W. (2019). Fusing character strengths and mindfulness interventions: Benefits for job satisfaction and performance. Journal of occupational health psychology, 24(1), 150-162. 1076-8998/19/\$12.00

Peterson, C., \& Park, N. (2006). Character strengths in organizations. Journal of Organizational Behavior, 27, 1149-1154. doi:10.1002/job.398

Peterson, C., \& Seligman, M. E. P. (2004). Character strengths and virtues: A handbook and classification. Washington: American Psychological Association. Seligman, M. E. P., \& Csikszentmihalyi, M. (2000). Positive psychology: An introduction. American Psychologist, 55(1), 5-14. 10.1037/0003-066X.55.1.

Tehranchi, A., Neshat Doost, H. T., Amiri, S., \& Power, M. J. (2018). The role of character strengths in depression: A structural equation model. Frontiers in Psychology, 9, 1609. 10.3389/fpsyg.2018.01609

Vazquez, A. C. S., Ferreira, M. C., \& Mendonça, H. (2019). Avanços na Psicologia Positiva: Bem-Estar, Engajamento e Redesenho no Trabalho. Avaliação Psicológica, 18(4), 343-351. 10.15689/ap.2019.1804.18859.02

Vianna, C. R. G. V. (2016). Mulheres na liderança: percepção e práticas do uso de forças de caráter em ambiente organizacional. In A. P. Correa (Org.), Psicologia Positiva: teoria e prática (pp. 172-180). Leader.

Yan, T., Chan, C. W. H., Chow, K. M., Zheng, W., \& Sun, M. (2020). A systematic review of the effects of character strengths-based intervention on the psychological well-being of patients suffering from chronic illnesses. Journal of Advanced Nursing. 10.1111/jan.14356 